\title{
Chemotherapy-induced Peripheral Neuropathy Among Paediatric Oncology Patients
}

\author{
M.J. Purser, D.L. Johnston, H.J. McMillan
}

\begin{abstract}
Background: Vinca alkaloids and platinum-containing chemotherapeutic drugs have the potential to cause chemotherapy-induced peripheral neuropathy (CIPN). This study determined the frequency of CIPN among children who were treated for acute lymphoblastic leukemia (ALL), lymphoma, brain tumour or Wilms tumour. Procedure: This retrospective cohort study reviewed 252 patients treated at the Children's Hospital of Eastern Ontario from 2001-2011. Patients were considered to have CIPN if they developed clinical symptoms of CIPN such as limb paraesthesia, weakness and/or ataxia during chemotherapy and their treating neurologist or oncologist deemed that their symptoms were due to a peripheral cause. Patients were excluded if their treatment regime did not include chemotherapy. Results: The overall frequency of CIPN was 18.3\% (46/252). Tumour-specific CIPN rates were: $18.9 \%$ (29/154) for ALL; $9.4 \%$ (3/32) for lymphoma; $17.9 \%$ (5/28) for Wilms tumour; and 23.7\% (9/38) for brain tumour patients. Nerve conduction studies were completed for $17 \%$ of patients (all tumour types) and were abnormal in all but one patient. Among surviving CIPN patients (41/46), 93\% showed no clinical deficits at their last examination, which was on average 56 months from time of diagnosis to last follow-up visit. Conclusions: The frequency of CIPN was less than that previously reported in adults receiving chemotherapy. Children with CIPN have a favourable outcome with most showing clinical improvement during the maintenance phase of treatment or after chemotherapy completion.
\end{abstract}

RÉSUMÉ: Neuropathie périphérique induite par la chimiothérapie chez des patients traités en oncologie pédiatrique. Contexte : Les vincaalkaloïdes et les médicaments contenant du platine utilisés en chimiothérapie ont le potentiel de causer une neuropahtie périphérique induite par la chimiothérapie (NPIC). Le but de l'étude était de déterminer la fréquence de la NPIC chez les enfants traités pour une leucémie lymphoblastique aiguë (LLA), un lymphome, une tumeur cérébrale ou une tumeur de Wilms. Méthode : Au cours de cette étude de cohorte rétrospective, les dossiers de 252 patients traités au Children's Hospital of Eastern Ontario (CHEO) entre 2001 et 2011 ont été revus. Les patients étaient considérés comme ayant une NPIC s'ils avaient présenté des symptômes cliniques de NPIC tels des paresthésies des membres, de la faiblesse et/ou de l'ataxie pendant la chimiothérapie et si le neurologue ou l'oncologue qui les traitait a jugé que leurs symptômes étaient dus à une cause périphérique. Les patients étaient exclus si leur schéma thérapeutique n'incluait pas de chimiothérapie. Résultats : La fréquence globale de NPIC était de 18,3\% (46/252). Les taux de NPIC spécifiques pour chaque tumeur étaient les suivants : 18,9\% (29/154) tous types de tumeurs confondus ; 9,4\% (3/32) pour les lymphomes ; 17,9\% (5/28) pour les tumeurs de Wilms et 23,7\% (9/38) pour les tumeurs cérébrales. Des études de conduction nerveuse ont été effectuées chez 17\% des patients (tous types de tumeurs confondus) et étaient anormales chez tous sauf un patient. Parmi les patients atteints de NPIC survivants (41/46), 93\% ne présentaient aucun déficit clinique au moment de leur dernier examen effectué en moyenne 56 mois après le diagnostic. Conclusions : La fréquence de la NPIC était inférieure à celle rapportée antérieurement chez des adultes recevant de la chimiothérapie. Les enfants atteints de NPIC ont une issue favorable et la plupart présentent une amélioration clinique au cours de la phase de traitement d'entretien ou après la fin de la chimiothérapie.

Can J Neurol Sci. 2014; 41: 442-447

Chemotherapy is responsible for improved survival rates of cancer patients although its use can be associated with neurotoxic side-effects including chemotherapy-induced peripheral neuropathy (CIPN). Certain chemotherapeutic agents have a higher predilection for peripheral nerve injury including: vincristine, vinblastine, cisplatin, taxanes, bortezomib and thalidomide ( $\mathrm{see}^{1}$ for review). Vincristine (VCR) and cisplatin are widely used in the treatment of patients with acute lymphoblastic leukemia (ALL), lymphoma, Wilms and brain tumours.

Chemotherapy-induced peripheral neuropathy generally manifests in a length-dependent manner. Chemotherapy-induced peripheral neuropathy symptoms can include distal limb paraesthesia and/or pain, distal muscle weakness, wasting and contractures. The frequency of CIPN among paediatric ALL patients has been estimated to be anywhere from $4 \%{ }^{2}$ to almost $35 \%{ }^{3}$ depending upon the diagnostic criteria used and the patient population and chemotherapeutic agents studied. Chemotherapyinduced peripheral neuropathy is more frequent among adults

From the University of Ottawa (MJP, DLJ, HJM) and Children's Hospital of Eastern Ontario (DLJ, HJM), Ottawa, Ontario, Canada.

Received October 3, 2013. Final Revisions Submitted February 5, 2014. Correspondence to: Hugh J. McMillan, Division of Neurology, Children's Hospital of Eastern Ontario, 401 Smyth Road, Ottawa, Ontario, K1H 8L1, Canada. Email: hmcmillan@cheo.on.ca. 
receiving chemotherapy, ranging from 30 to $75 \%$ of patients ${ }^{4,5}$. Several factors have been associated with a higher incidence of CIPN including: pre-existing neuropathy (e.g. diabetes), prior or concomitant treatment with other neurotoxic agents, as well as personal factors that can include decreased CYP3A5 expression 6 . Other factors including the total dose of VCR administered is of unclear significance. Some studies have identified an association between CIPN and VCR dose while others have $\operatorname{not}^{7,8}$.

The goal of this study was to determine the frequency of symptomatic CIPN in a cohort of paediatric oncology patients receiving chemotherapy at a Canadian tertiary care paediatric hospital. Children treated for ALL, lymphoma, Wilms or brain tumours were included due to the use of CIPN-related chemotherapeutic agents in the therapy for these cancers. Their presenting symptoms, disease severity and outcome were recorded.

\section{Methods And Materials}

Institutional research ethics board approval was obtained prior to the start of data collection. The medical records of all patients with ALL, lymphoma, brain tumours and Wilms tumour who received chemotherapy at the Children's Hospital of Eastern Ontario (CHEO) Haematology-Oncology Clinic from 20012011 were reviewed.

\section{Inclusion/Exclusion Criteria}

Inclusion criteria included: 1 ) age $\leq 18.0$ years old at the time of cancer diagnosis; 2) tissue diagnosis confirmed by pathology, haematopathology, or imaging; 3 ) chemotherapy used to treat the underlying disease; 4) medical records available for review. Exclusion criteria included: 1) treatment regime did not include chemotherapy; 2) incomplete medical records available for review and; 3) imaging that identified a radiologically-defined cause for the child's neurological symptoms other than peripheral neuropathy.

\section{Clinical Data}

Patients' medical charts were reviewed and the following information extracted: 1) gender; 2) age at cancer diagnosis; and 3) cancer type, subtype and/or risk stratification. Patients with ALL were classified by type (pre-B-cell, mature B-cell or T-cell) and risk (low, standard or high). Lymphoma patients were classified by microscopic appearance and immunophenotype (Bcell, T-cell, Hodgkin or other). Brain tumour patients were classified by tumour type and graded as per the World Health Organization grading system. Wilms tumour patients were categorized by stage $(1,2,3,4$, or 5$)$. Additional information collected included patients': 4) symptoms at disease presentation; 5) age at chemotherapy initiation; 6) chemotherapy agents used; 7) whether or not radiation therapy was employed (including radiation site and dose); 8) whether or not a disease relapse occurred (including time and site of relapse site as well as subsequent treatment); and 9) medical history, including comorbid diagnoses, neurodevelopment and family medical history.

Medical charts were reviewed to determine if patients showed any clinical symptoms of CIPN during chemotherapy.
Symptoms could include sensory complaints of paraesthesia, dysesthesia and/or ataxia, as well as muscle weakness due to foot drop, or hand weakness. Clinical signs of neuropathy on physical examination such as sensory loss, muscle weakness, hyporeflexia, areflexia or dyscoordination were noted. Electrodiagnostic test results (e.g. nerve conduction study (NCS) and electromyography (EMG) were noted. Diagnostic imaging results magnetic resonance imaging (MRI) brain and spine were reviewed.

\section{Definition of CIPN}

Patients were considered to have CIPN if they developed symptoms of sensory and/or motor impairment during chemotherapy that their oncologist or neurologist attributed to a peripheral nerve lesion. Patients were not classified as CIPN if they showed clinical features and/or neuroimaging findings consistent with a central (brain or spine) cause for their symptoms. Patients were considered to have CIPN if their sensory and/or motor symptoms resulted in functional impairment, thus corresponding to a Common Toxicity Criteria Adverse Events (CTCAE) Grade 2 or higher toxicity ${ }^{9}$. The CTCAE grades for peripheral neuropathy are defined as: Grade 1 = asymptomatic; loss of deep tendon reflexes and/or mild paraesthesia; Grade 2 = moderate symptoms affecting activities of daily living; Grade 3 = severe symptoms; limiting self-care and/or necessitating assistive devices; Grade 4 = life-threatening consequences and urgent intervention indicated; Grade $5=$ death. For patients with progressive symptoms, the grade corresponding to their worst clinical symptoms that were documented was applied.

\section{Statistical Analysis}

Data was analyzed using GraphPad Prism software (GraphPad Software, Inc., San Diego, CA, USA). Statistical analysis was performed using unpaired, two-tailed student t-test, and significance was determined with $\mathrm{p} \leq 0.05$.

\section{RESUlts}

There were 274 patients included in this study who were treated at the CHEO Haematology-Oncology Clinic from 20012011. Specific diagnoses included: ALL ( $=168)$, lymphoma $(\mathrm{N}=35)$, brain tumour $(\mathrm{N}=38)$ or Wilms tumour $(\mathrm{N}=33)$. Of these patients, 252/274 (92\%) met inclusion criteria. Of the 22 patients who were excluded: ten had incomplete records (e.g. chemotherapeutic treatment was initiated at a different hospital or the patient moved away during treatment), eight were found to have a central cause responsible for their neuropathy symptoms (e.g. methotrexate leukoencephalopathy or spinal metastases causing compression), and four patients received treatment that did not include any chemotherapy.

\section{Demographic Information}

The gender of the 252 eligible patients for this study included 133 males (76 ALL, 21 lymphoma, 26 brain tumour, 10 Wilms tumour) and 119 females (78 ALL, 11 lymphoma, 12 brain tumour, and 18 Wilms tumour). No patients had any personal or family history of Charcot-Marie-Tooth disease or other inherited neuropathies. The average age at cancer diagnosis was: 6.7 years 
Table 1: Frequency and severity of chemotherapy-induced peripheral neuropathy (CIPN) Common Toxicity Criteria Adverse Event (CTCAE) Grade ${ }^{9}$ for each cancer type

\begin{tabular}{|c|c|c|c|c|c|}
\hline \multirow[t]{2}{*}{ Cancer type } & \multirow{2}{*}{$\begin{array}{c}\text { CIPN } \\
\text { Frequency (\# patients) }\end{array}$} & \multirow[b]{2}{*}{ Gr2 } & \multicolumn{3}{|c|}{ CTCAE Grade } \\
\hline & & & Gr3 & Gr4 & Gr5 \\
\hline ALL & $18.8 \% \quad(29 / 154)$ & 27 & 2 & & \\
\hline Lymphoma & $(3 / 32)$ & 2 & 1 & & \\
\hline Brain tumour & $(9 / 38)$ & 4 & 5 & & \\
\hline Wilms tumour & $(5 / 28)$ & 3 & 2 & & \\
\hline Overall & $18.3 \% \quad(46 / 252)$ & 36 & 10 & 0 & 0 \\
\hline
\end{tabular}

for ALL patients, 10.8 years for lymphoma patients, 6.4 years for brain tumour patients and 3.5 years for Wilms tumour patients.

\section{Cancer type \& subtype / risk stratification}

Among ALL patients $(\mathrm{N}=154) ; 131$ patients had precursor $\mathrm{B}-$ cell ALL (46 high risk, 67 standard risk, 18 low risk); 16 had Tcell ALL (6 high risk); 7 had mature B-cell (Burkitt's) leukemia. Among lymphoma patients $(\mathrm{N}=32) ; 7$ had lymphoblastic lymphoma, 11 had B-cell lymphoma, 1 had T-cell lymphoma, 2 had anaplastic large cell lymphoma, and 11 had Burkitt's lymphoma. Among the brain tumour patients $(\mathrm{N}=38)$; 20 had medulloblastoma (12 high risk), 11 had astrocytoma, 3 had germ cell tumours, 2 had atypical teratoid/rhabdoid tumour, 1 had embryonal carcinoma of the thalamus, and 1 patient had a supratentorial primitive neuroectodermal tumour. Among Wilms tumour patients $(\mathrm{N}=28)$ the staging varied from Stage $1(\mathrm{~N}=7), 2$ $(\mathrm{N}=5), 3(\mathrm{~N}=4), 4(\mathrm{~N}=8)$ or $5(\mathrm{~N}=4)$. No difference was noted between cancer types or subtypes among patients who developed CIPN compared to those who did not.

\section{Frequency of CIPN}

The overall frequency of symptomatic CIPN (CTCAE Grade 2 or higher) at our centre was $18.3 \%(46 / 252)$. The frequency for specific cancer type was: ALL $18.8 \%$ (29/154); lymphoma $9.4 \%$ $(3 / 32)$; brain tumour $23.7 \%(9 / 38)$ and Wilms tumour $17.9 \%$
(5/28). Most patients with CIPN had Grade 2 toxicity (Table 1). Patients with Grade 3 toxicity had a reduction in chemotherapy dose. Most patients with CIPN presented with either sensorimotor symptoms (39\% patients) or motor-only symptoms (46\%; Table 2) with a minority (15\%) presenting with sensory-only symptoms. Acute lymphoblastic leukemia patients showed a similar frequency of initial CIPN symptoms. Among ALL patients who presented with sensorimotor or motor symptoms, foot drop was noted in $82.7 \%$ (24/29). Other initial CIPN symptoms included ataxia/clumsy gait $(45 \% ; 13 / 29)$ and impairment of fine motor tasks $(24.1 \% ; 7 / 29)$. Of the $17 \%$ ALL patients with sensory CIPN symptoms patients complained of either paraesthesia causing gait impairment or severe limb dysesthesia. All lymphoma patients with CIPN presented with sensorimotor symptoms including foot-drop and limb paraesthesia or pain. Among brain tumour patients with CIPN, $56 \%(5 / 9)$ presented with foot drop with or without hand weakness. One-third presented with sensorimotor symptoms (foot drop and severe limb paraesthesia or pain). A four year-old patient receiving treatment for a posterior fossa astrocytoma developed progressive gait ataxia in the final months of chemotherapy. Her clinical exam noted intact muscle strength and decreased or absent deep tendon reflexes. Formal sensory testing was deemed unreliable due to young age and poor cooperation. Her MRI brain showed no disease progression. The patient did not undergo NCS which could have helped

Table 2: Clinical symptoms of children who developed CIPN

\begin{tabular}{lccc}
\hline & & Clinical symptoms \\
Cancer type & Sensorimotor & Motor-only & Sensory-only \\
\cline { 2 - 3 } & & & \\
ALL & $38 \%(11 / 29)$ & $45 \%(13 / 29)$ & $17 \%(5 / 29)$ \\
Lymphoma & $100 \%(3 / 3)$ & $60 \%(3 / 5)$ & $10 \%(1 / 5)$ \\
Wilms tumour & $20 \%(1 / 5)$ & $56 \%(5 / 9)$ & $11 \%(1 / 9)$ \\
Brain tumour & $33 \%(3 / 9)$ & $46 \%(21 / 46)$ & $15 \%(7 / 46)$ \\
Overall & $39 \%(18 / 46)$ & & \\
\hline
\end{tabular}


differentiate a length-dependent sensory neuropathy from a nonlength dependent ganglionopathy. She remained in remission at the time of last clinical follow-up, four years post-chemotherapy. Her symptoms improved with no clinical concerns documented in her last follow-up visit. Among Wilms tumour patients, $60 \%$ presented with foot drop with or without hand weakness. One had foot drop and severe lower extremity dysthesesia. One presented with severe lower extremity dysesthesia and areflexia without weakness.

Confirmatory electrodiagnostic testing has not been routinely completed for children with suspected CIPN at our centre. Only $17 \%(8 / 46)$ patients with suspected CIPN had NCS and EMG. Of these patients, all but one showed NCS evidence for a sensorimotor polyneuropathy with axonal features. The one patient with a normal NCS presented with severe limb dysesthesia without weakness suggesting that small unmyelinated pain fibres were predominantly or exclusively involved. Since NCS preferentially measures large myelinated sensory fibers, this technique would not be able to detect a disorder affecting this fibre type.

Chemotherapy and radiotherapy use was recorded for each cancer (Table 3). No significant difference was noted in the use of vincristine among children who did develop CIPN compared to those who did not. Radiation therapy was used in $13 \%$ (20/154) of children treated for ALL. Chemotherapy-induced peripheral neuropathy was diagnosed in a minority of children who received radiotherapy for ALL $(25 \% ; 5 / 20)$, Wilms tumour $(23 \% ; 3 / 13)$ and brain tumours $(28 \% ; 7 / 25)$. Among lymphoma patients receiving radiotherapy, CIPN was noted in a higher proportion $(50 \% ; 2 / 4)$ however sample size was small for this group. No patients were noted to have fasciculations or asymmetrical symptoms that might point to a radiation neuritis or plexitis.

\section{Long-term outcome}

Long-term clinical follow-up was favourable for most CIPN patients. Among ALL patients with CIPN 28/29 were alive at follow-up; 18 patients were off-treatment, ten patients were still receiving maintenance chemotherapy and one patient was deceased (acute ischemic stroke associated with radiation vasculitis). Of the 28 surviving patients, $89.3 \%$ (25/28) had no CIPN symptoms at the time of last clinical follow-up, which was an average of 56 months from time of diagnosis. The three patients with ongoing CIPN symptoms had persistent foot drop, leg weakness and/or gait impairment at the time of last clinical follow-up, which for these three patients was an average of one year after chemotherapy was completed. Two of these patients had been classified as having CTATE Grade 3 peripheral neuropathy. Disease relapse occurred in 20/154 ALL patients, four of whom were diagnosed with CIPN.

All lymphoma patients with CIPN and all but one Wilms tumour patient with CIPN were alive at last follow-up, which was an average of 70 and 46 months from time of diagnosis, respectively. None of the surviving lymphoma or Wilms tumour patients had symptoms of CIPN at last follow-up. Two lymphoma patients relapsed, neither of whom were diagnosed with CIPN. Disease relapse occurred in $4 / 28$ Wilms tumour patients, one of whom had CIPN.

Among brain tumour patients with CIPN, 6/9 were alive with no symptoms of CIPN apparent at last follow-up, which was an average of 56 months from time of diagnosis. Two of the surviving brain tumour patients required a reduction in vincristine dose during therapy. Four of the six living brain tumour patients who had CIPN have mild-to-moderate sensorineural hearing loss. Disease relapse occurred in $8 / 38$ brain tumour patients of whom two had CIPN.

\section{Discussion}

Chemotherapy induced peripheral neuropathy was seen in $18.3 \%$ of children receiving treatment for ALL, lymphoma, brain and Wilms tumour at our institution with the highest frequency seen among brain tumour patients. Given that this was a retrospective study our results are likely to be a conservative estimate of CIPN frequency. Some children with mild CIPN

Table 3: Chemotherapeutic agents used in paediatric oncology patients

\begin{tabular}{|c|c|}
\hline Cancer type & Chemotherapy and radiotherapy use (\% patients receiving treatment) \\
\hline $\begin{array}{l}\text { ALL } \\
(\mathrm{N}=154)\end{array}$ & $\begin{array}{l}\text { vincristine }(100 \%) \text {, corticosteroids }(100 \%) \text {, methotrexate }(99 \%) \text {, asparaginase }(95 \%) \text {, } \\
\text { mercaptopurine }(95 \%) \text {, cytarabine }(90 \%) \text {, doxorubin or daunorubicin }(79 \%) \text {, } \\
\text { cyclophosphamide }(63 \%) \text {, thioguanine }(63 \%) \text {, radiotherapy* }(13 \%)\end{array}$ \\
\hline $\begin{array}{l}\text { Lymphoma } \\
(\mathrm{N}=32)\end{array}$ & $\begin{array}{l}\text { doxorubicin or daunorubicin }(94 \%) \text {, vincristine }(91 \%) \text {, cyclophosphamide }(84 \%) \text {, } \\
\text { methotrexate }(78 \%) \text {, cytarabine }(75 \%) \text {, mercaptopurine }(31 \%) \text {, rituximab }(35 \%) \text {, } \\
\text { radiotherapy* }(13 \%)\end{array}$ \\
\hline Wilms tumour $(\mathrm{N}=28)$ & vincristine $(100 \%)$, dactinomycin $(89 \%)$, doxorubicin $(64 \%)$ radiotherapy* $(46 \%)$ \\
\hline Brain tumour $(\mathrm{N}=38)$ & $\begin{array}{l}\text { vincristine }(79 \%) \text {, cisplatin }(65 \%) \text {, cyclophosphamide }(50 \%) \text {, carboplatin }(32 \%) \text {, } \\
\text { radiotherapy* }(66 \%)\end{array}$ \\
\hline
\end{tabular}

*Local, cranial or craniospinal radiotherapy was used. 
symptoms may have been missed. Moreover, our definition of CIPN did not include children who were asymptomatic (e.g. isolated areflexia) or who had an isolated autonomic neuropathy (e.g. constipation) as we deemed these symptoms less clinically relevant since they do not trigger a reduction of chemotherapy dose. All brain tumour patients are followed by a dedicated paediatric neurologist and paediatric oncologist at our hospital. Complete serial neurological examinations were completed for all brain tumour patients. Children with ALL, lymphoma and Wilms tumour are followed by a team of pediatric oncologists and referred to one of two paediatric neurologists when CIPN or other neurological symptoms were noted.

Vincristine can cause a mixed sensorimotor polyneuropathy. Vincristine related CIPN begins with loss of deep tendon reflexes at the ankles progressing to more widespread areflexia with distal sensory fibre involvement ${ }^{5,10}$. Muscle spindle afferents are thought to be the first sign of CIPN since H-reflexes are initially intact in these areflexic patients ${ }^{10}$. The H-reflex is the electrophysiological equivalent of the ankle deep tendon reflex, utilizing the same sensory and motor pathways except for the muscle spindle afferents that initiate this physiological response. Over time CIPN progresses to become a lengthdependent axonal neuropathy ${ }^{10}$. Other chemo-therapeutic agents such as cisplatin and carboplatin are used in the treatment of brain tumours and have a higher predilection for causing a sensory neuropathy ${ }^{11}$. All four of the brain tumour patients with a sensory neuropathy received either cisplatin or carboplatin as part of their treatment. Given that brain tumour patients were found to have the highest rate of CIPN and that cisplatin and carboplatin were the only chemotherapeutic agents unique to this group this may warrant future consideration as to potential cumulative effects of multiple CIPN-causing drugs in this group. The risk of VCR-related CIPN in adults is believed to occur in dose-dependent manner with higher cumulative VCR exposure increasing the likelihood of CIPN ${ }^{7}$. This relationship is less clear among children receiving VCR with one prospective study showing motor difficulties to be independent of cumulative VCR dosing ${ }^{8}$. Recently, increasing attention is being paid to variability of individual patient drug pharmacokinetics. Adult cancer patients have been found to have wide inter-individual variation in VCR metabolism resulting in an 11-fold variation in the dosecorrected area under the drug concentration-time curve ${ }^{12}$. Similar variability has been demonstrated in children treated with $\mathrm{VCR}^{13}$. Such studies indicate that while VCR dose may contribute to CIPN, there is likely a more complex relationship between genetic variability in cytochrome $\mathrm{P} 450$ pathways among individual cancer patients. Genetic variation of CYP3A5 has been associated with a lower risk of VCR-related CIPN in a population of paediatric ALL patients ${ }^{6}$. Concomitant use of other medications (e.g. fluconazole) may further alter P450 metabolism and thus the clearance of chemotherapeutic agents. We did not collect information on the use of these medications. However, standard practice at our hospital is to hold these medications for 24 hours before and 24 hours after any dose of chemotherapy to minimize this risk.

Neuropathic pain has been reported in as high as $35 \%$ of ALL patients ${ }^{14}$. No relationship has been identified between the pain severity and the cumulative dose of VCR administered, although some patients experience intermittent and recurrent pain during treatment ${ }^{14}$. Most of the children with CIPN at our centre who presented with sensory symptoms complained of severe limb dysesthesia which responded well to treatment with gabapentin and/or opioids. Pain symptoms had resolved in all cases by the time of last follow-up.

Most children with CIPN show relatively mild symptoms ${ }^{15}$ as was the case at our centre where most showed CTCAE Grade 2 (Table 2). No children at our centre had any personal or family history of inherited neuropathies which is an important item on history since patients with Charcot-Marie-Tooth can suffer from a rapid-onset and severe debilitating polyneuropathy from exposure to $\mathrm{VCR}^{16}$.

Electrodiagnostic testing was performed in a small percentage of children with CIPN at our centre. We hypothesize that this may have been due to their relatively mild CIPN in most cases, as well as a desire of parents and caregivers to avoid what is often perceived to be an uncomfortable diagnostic test. We favour the completion of NCS in children who are suspected to have CIPN and recommend that testing be done in conjunction with another sedated procedure if the child is not anticipated to be capable of tolerating the study. In some instances, parents and caregivers may focus upon completion of chemotherapy and ignore treatment related side-effects until they become more troublesome. This may be particularly true for children who are nearing the end of chemotherapy. We believe that NCS are important to accurately diagnose and monitor CIPN severity and progression. It is an important testing modality to confirm peripheral nerve involvement and rule out lesions elsewhere in the neuraxis.

Given the retrospective nature of our study there are several inherent limitations. Our study may have underestimated the frequency of CIPN, particularly in young children or those with milder CIPN symptoms. Another limitation is the fact that the majority of clinical examinations for patients with ALL, lymphoma and Wilms tumour were completed by nonneurologists. As such there may be a reduced sensitivity for detecting subtle neurological deficits when present. This again could underestimate the frequency of CIPN and/or create the impression of a more favorable long-term outcome. Another limitation of our study is that our study was designed to identify children with possible CIPN based upon predetermined clinical criteria and to then exclude those identified with a radiologically-defined cause for their neurological condition (e.g. leukoencephalopathy). Since only a minority of CIPN patients had a NCS performed we cannot completely exclude the possibility that some patients with diagnoses other than a peripheral nerve lesion (e.g. steroid myopathy, radiation-induced myelitis) may have been inadvertently included. This limitation underscores the importance of electrodiagnostic testing in confirming CIPN among paediatric cancer patients.

Historically, neurotoxicity has been poorly documented in the oncology literature with some studies lumping central and peripheral abnormalities together ${ }^{2}$. This is particularly problematic in pediatric oncology and can make an accurate literature review and counseling difficult. Neuropathy symptoms are being increasingly recognized in long-term survivors of ALL. Most children with CIPN at our centre demonstrated a favourable outcome at the time of last clinical assessment. However, a large follow-up study of 415 children who received 
chemotherapy for ALL found almost $13 \%$ of long-term survivors reported neuropathic symptoms (e.g. numbness, tingling, fine motor impairment $)^{17}$. Another smaller study reported lower limb hyporeflexia, fine motor and/or gross motor deficits in 33\% of children even five years after completion of chemotherapy ${ }^{18}$. Such observations underscore the need for surveillance studies of children receiving chemotherapy in order to adequately monitor the onset and progression of CIPN. Such surveillance could include a combination of standardized physiotherapy assessments, patient questionnaires and serial nerve conduction studies while the patient is sedated for other tests. Data pertaining to patients' genotypic variables that may alter pharmacokinetics of chemotherapy drugs as well as the concomitant use of other medications that could alter P450 metabolism should also be considered in any prospective study. Such data would be crucial for a well-designed CIPN natural history study, prevention study or treatment trial.

In conclusion, this is one of only a few studies that have reported the frequency of CIPN among paediatric oncology patients. We have documented a variable rate of CIPN among children treated for ALL, lymphoma, brain or Wilms tumour and have provided a detailed description of the 252 patients at our centre in an attempt to document potential markers that could be used to better predict CIPN occurrence, treatment efficacy, and/or patient outcomes. We report a favourable long-term outcome for paediatric survivors of cancer diagnosed with CIPN.

\section{ACKNOWLEDGEMENTS}

The authors thank the Leukemia and Lymphoma Society of Canada for funding a summer student bursary for MJP. These findings were presented at the 48th Annual Congress of the Canadian Neurological Sciences Federation, June 11-14, 2013 in Montréal, Québec and the 26th Annual Meeting of the American Society of Pediatric Hematology/Oncology, April 24-27, 2013 in Miami, Florida.

\section{REFERENCES}

1. Gilchrist L. Chemotherapy-induced peripheral neuropathy in pediatric cancer patients. Semin Pediatr Neurol. 2012;19(1): 9-17.

2. Aytac S, Yetgin S, Tavil B. Acute and long-term neurologic complications in children with acute lymphoblastic leukemia. Turk J Pediatr. 2006;48(1):1-7.

3. Renbarger JL, McCammack KC, Rouse CE, Hall SD. Effect of race on vincristine-associated neurotoxicity in pediatric acute lymphoblastic leukemia patients. Pediatr Blood Cancer. 2008;50 (4):769-71.

4. Wolf S, Barton D, Kottschade L, Grothey A, Loprinzi C. Chemotherapy-induced peripheral neuropathy: prevention and treatment strategies. Eur J Cancer. 2008;44(11):1507-15.

5. Pal PK. Clinical and electrophysiological studies in vincristine induced neuropathy. Electromyogr Clin Neurophysiol. 1999;39 (6):323-30.

6. Egbelakin A, Ferguson MJ, MacGill EA, et al. Increased risk of vincristine neurotoxicity associated with low CYP3A5 expression genotype in children with acute lymphoblastic leukemia. Pediatr Blood Cancer. 2011;56(3):361-7.

7. Verstappen CC, Koeppen S, Heimans JJ, et al. Dose-related vincristine-induced peripheral neuropathy with unexpected offtherapy worsening. Neurology. 2005;64(6):1076-7.

8. Hartman A, van den Bos C, Stijnen T, Pieters R. Decrease in motor performance in children with cancer is independent of the cumulative dose of vincristine. Cancer. 2006;106(6):1395-401.

9. U.S. Department of Health and Human Services [homepage on the internet]. Common Terminology Criteria for Adverse Events (CTCAE) Version 4.0 [updated 2010 June 14; cited 2013 Sep 17]. Available from: http://evs.nci.nih.gov/ftp1/CTCAE/ CTCAE_4.03_2010-06-14_QuickReference_8.5x11.pdf

10. Casey EB, Jellife AM, Le Quesne PM, Millett YL. Vincristine neuropathy. Clinical and electrophysiological observations. Brain. 1973;96(1):69-86.

11. Sghirlanzoni A, Silvani A, Scaioli V, Pareyson D, Marchesan R, Boiardi A. Cisplatin neuropathy in brain tumor chemotherapy. Ital J Neurol Sci. 1992;13(4):311-15.

12. Van den Berg HW, Desai ZR, Wilson R, Kennedy G, Bridges JM, Shanks RG. The pharmacokinetics of vincristine in man: reduced drug clearance associated with raised serum alkaline phosphatase and dose-limited elimination. Cancer Chemother Pharmacol. 1982;8(2):215-19.

13. Gidding CE, Meeuwsen-de Boer GJ, Koopmans P, Uges DR, Kamps WA, de Graaf SS. Vincristine pharmacokinetics after repetitive dosing in children. Cancer Chemother Pharmacol. 1999;44(3):203-9.

14. Anghelescu DL, Faughnan LG, Jeha S, et al. Neuropathic pain during treatment for childhood acute lymphoblastic leukemia. Pediatr Blood Cancer. 2011;57(7):1147-53.

15. Reinders-Messelink HA, Van Weerden TW, Fock JM, et al. Mild axonal neuropathy of children during treatment for acute lymphoblastic leukaemia. Eur J Paediatr Neurol. 2000;4(5): 225-33.

16. Chauvenet AR, Shashi V, Selsky C, Morgan E, Kurtzberg J, Bell B. Vincristine-induced neuropathy as the initial presentation of charcot-marie-tooth disease in acute lymphoblastic leukemia: a Pediatric Oncology Group study. J Pediatr Hematol Oncol. 2003; 25(4):316-20.

17. Ness KK, Hudson MM, Pui CH, et al. Neuromuscular impairments in adult survivors of childhood acute lymphoblastic leukemia: associations with physical performance and chemotherapy doses. Cancer. 2012;118(3):828-38.

18. Lehtinen SS, Huuskonen UE, Harila-Saari AH, Tolonen U, Vainionpaa LK, Lanning BM. Motor nervous system impairment persists in long-term survivors of childhood acute lymphoblastic leukemia. Cancer. 2002;94(9):2466-73. 